\title{
Aceptabilidad y adopción de guías de práctica clínica y protocolos de atención sobre preeclampsia-eclampsia en la República Dominicana
}

\author{
Andelys De la Rosa' ${ }^{1}$, José Mordan², Indiana Barinas ${ }^{1}$, Mayra Toribioº , Diana Mancebo ${ }^{1}$, \\ Alexandra Rodríguez ${ }^{3}$ y Mar Pacheco-Herrero 4
}

Forma de citar

De La Rosa A, Mordan J, Barinas I, Toribio M. Mancebo D, Rodríguez A, et al. Aceptabilidad y adopción de guías de práctica clínica y protocolos de atención sobre preeclampsia-eclampsia en la República Dominicana. Rev Panam Salud Publica. 2020;44:e163. https://doi.org/10.26633/RPSP.2020.163

RESUMEN

Objetivo. Estimar la aceptabilidad y adopción de las guías de prática clínica (GPC) y protocolos de atención a la mujer con preeclampsia-eclampsia por parte del personal prestador de los servicios de salud, e identificar los factores facilitadores y las barreras para su implementación.

Métodos. Se desarrolló un estudio cualitativo por medio de entrevistas semiestructuradas y grupos focales en cinco maternidades. Se recopilaron las entrevistas para su análisis y se caracterizaron las barreras y facilitadores.

Resultados. Participaron 70 profesionales de la salud (52 de sexo femenino y 18 de sexo masculino) que se desempeñan en distintos niveles del sistema de salud. La mayoría de los prestadores y gerentes conocen la existencia de las GPC de eclampsia-preeclampsia y su contenido, sobre todo los participantes con más tiempo en el servicio. Para los facilitadores, se estableció una valoración positiva entre el personal médico y de enfermería ante el proceso de continuar con la elaboración e implementa-ción de GPC de alta calidad. Hubo consenso en cuanto a la existencia de limitaciones, sobre todo, por la falta de medicamentos, insumos y equipos requeridos, para cumplir y aplicar las recomendaciones formuladas.

Discusión. Los resultados del estudio exponen la necesidad de fortalecer estrategias que ayuden a cerrar la brecha entre la investigación y la política pública. Estudios fundamentan la investigación en priorizar la atención a los usuarios, y los encargados de formular políticas y los tomadores de decisiones en el sistema de salud. Los actores del sistema de salud dominicano reconocen la metodología GRADE como un instrumento apropiado para la formulación e implementación de GPC. Las barreras de implementación requieren de abordajes sistémicos e integrales.

Palabras clave Guía de práctica clínica; protocolos; aceptabilidad de la atención de salud; políticas de salud; República Dominicana.

\footnotetext{
1 Ministerio de Salud Pública y Asistencia Social, República Dominicana. $\triangle$ Andelys De la Rosa, andelysdelarosa@gmail.com,

2 Consultores independientes.
}

3 Organización Panamericana de la Salud, República Dominicana. Caballeros, República Dominicana. 
Los esfuerzos por dotar a los sistemas de salud de recursos para mejorar la prestación de los servicios clínicos han propiciado el desarrollo de directrices llamadas guías de práctica clínica (GPC), protocolos o normas clínicas informadas por evidencias, que aportan pruebas sólidas sobre la eficacia, la seguridad y los costos de las intervenciones sanitarias (1).

La calidad de los instrumentos metodológicos utilizados para la formulación de las GPC y directrices en el campo de la salud se sustenta en la sistematización de la información científica y la adopción de parámetros explícitos con el objetivo de aportar pruebas sólidas que apoyan las recomendaciones. En este orden, la metodología GRADE incorpora la revisión sistemática de la literatura, propone la formulación de tablas y perfiles de evidencias y se convierte así en un medio adoptado y promovido por la Organización Panamericana de la Salud (OPS), academias y otras organizaciones de investigación y publicación de prestigio global $(2,3)$.

La promoción de las guías informadas por evidencias mediante la metodología GRADE ha impulsado a los sistemas de salud de América Latina y el Caribe a invertir recursos técnicos y financieros en programas de formulación de GPC, directrices y protocolos con el decidido apoyo de la OPS en alianza con centros de investigación y equipos de expertos locales organizados en estructuras de especialidades clínicas (2). La República Dominicana también se ha integrado al proceso de mejora de la calidad de sus directrices para la atención clínica mediante el estímulo de procesos de formación de equipos humanos para formulación o adaptación de GPC con resultados relevantes en el campo de la atención maternoinfantil, de forma particular, en la prevención y atención de la morbilidad materna por preeclampsia y atención del recién nacido prematuro. En el campo de la atención a la morbilidad por preeclampsia-eclampsia, patología de mayor dimensión en términos de complicación y mortalidad materna en el país, se han desarrollado esfuerzos por introducir y sostener estrategias para su control. Estas incluyen la expansión de los controles prenatales, la formación gradual de más especialistas en el campo de la obstetricia, la instalación de unidades de cuidados intensivos maternos y mejoras a través de los cuidados obstétricos de emergencias, hechos que se traducen en resultados parciales e insuficientes cuya mejora continua constituye un desafío para el sistema de salud.

El dato que confirma la idea anterior es la razón de mortalidad materna informada de 104 muertes por cada 100000 nacidos vivos.

La preeclampsia-eclampsia es la causa más frecuente de mortalidad materna en el país. Este contexto justifica el fortalecimiento de las acciones que apoyen la implementación de las GPC, sobre todo, las guías basadas en las mejores pruebas, las de mayor aceptación y adoptadas por los servicios de salud y las organizaciones del sistema (4). En el 2019 se informaron 171 muertes maternas, sin informe de la razón de mortalidad materna (MM).

La investigación sobre la implementación de las GPC y los protocolos se plantea en el marco de transición de la Unidad Coordinadora de Guías y Protocolos hacia la categoría de Programa Nacional, proceso que facilitaría un mayor alcance territorial y el desarrollo de las competencias locales para apoyar la agenda de la calidad de atención. Para el logro de esta meta se identifica la necesidad de recopilar la información científica necesaria para analizar los obstáculos para su implementación.
El sistema de salud dominicano ha experimentado cambios estructurales: la separación de funciones, la expansión del sistema dominicano de seguridad social y el establecimiento de políticas enfocadas en prioridades, entre las que se destaca la necesidad de proteger a la población de mayor vulnerabilidad, brindar atención especial a los problemas de salud sexual y reproductiva y prevenir la mortalidad materna e infantil $(5,6)$.

La investigación sobre la implementación de las recomendaciones formuladas por los programas de salud es un componente crucial de los países mejorar la salud y los sistemas de salud, sobre todo en países de bajos y medianos ingresos. La OPS propone este enfoque de colaboración, propiciado a través de la iniciativa Embedded Implementation Research como un modelo innovador que involucra de forma directa a los responsables de políticas sanitarias en calidad de investigadores y los líderes de equipos locales. El objetivo es que, al momento de tomar decisiones en el campo sanitario, cuenten con información primaria que permita identificar y analizar los factores relacionados con el contexto y los determinantes de la implementación de las intervenciones, políticas o programas de salud (7).

En el caso de las áreas asistenciales más sensibles, como las relacionadas con la maternidad y el desarrollo infantil, resulta crucial analizar los factores que determinan la aceptabilidad y adopción de las GPC y los protocolos de atención, de forma particular, el contexto y organización de los servicios de atención materna e infantil, los conocimientos de los prestadores clínicos, la percepción de la calidad de las normas, la relevancia y la pertinencia de las directrices clínicas, los factores tecnológicos, los recursos financieros y las prácticas habituales, entre otros componentes moduladores de su implementación.

El objetivo principal de este estudio es estimar el grado de aceptabilidad y adopción de las GPC y protocolos de atención a la mujer con preeclampisa-eclampsia por parte de los prestadores d ellos servicios de salud e identificar los factores facilitadores y las barreras percibidas por los actores vinculados al proceso de elaboración de estas guías y protocolos.

\section{MATERIALES Y MÉTODOS}

La presente investigación se plantea en el marco de la iniciativa de la OPS y de la Alianza para la Investigación sobre Políticas y Sistemas de la Organización Mundial de la Salud para promover la implementación de intervenciones sanitarias con el fin de avanzar en la agenda que promueve la Cobertura Universal en Salud y los Objetivos de Desarrollo Sostenible (8). El diseño del estudio fue de tipo cualitativo, en el que se recogió información basada en la observación. Asimismo, se trabajó de una forma estratégica en la que, en términos prácticos, el proceso de implementación de políticas tiene como objetivo transferir las recomendaciones contenidas en las GPC al quehacer diario de la práctica clínica.

Las técnicas utilizadas fueron entrevistas semiestructuradas y grupos focales contenidos en cinco maternidades de referencia nacional, donde se presentan la mayor cantidad de casos de mortalidad materna, tres de ellas en hospitales docentes donde hay mayor influencia para el conocimiento y aplicación de las GPC. Las instituciones seleccionadas fueron: la Maternidad de Los Mina, con 10921 nacimientos al año y ubicada en Santo Domingo (capital del país); la Maternidad René Klan, con 7380 
nacimientos y ubicada en Santiago (segunda provincia con mayor cantidad de habitantes de la República Dominicana); la Maternidad Juan Pablo Pina de San Cristóbal, en la región sudoeste del país y con 4584 nacimientos; la Maternidad Jaime Sánchez, en la provincia de Barahona (zona fronteriza con Haití) y con 3986 nacimientos anuales; y el Hospital Antonio Musa de San Francisco de Macorís, ubicado en la región nordeste del país. Se recogió la perspectiva de los 70 prestadores durante el 2018.

La población participante estuvo integrada por 23 enfermeras, 12 médicos residentes de ginecología y obstetricia, 12 médicos especialistas en ginecobstetricia, 10 gerentes de salud y dos líderes formuladores, con un total de 52 participantes de sexo femenino y 18 de sexo masculino.

Se abarcaron distintos niveles del sistema de salud, donde se incluyeron autoridades, tomadores de decisiones, elaboradores de políticas públicas y funcionarios de organismos administradores de salud de distinta complejidad, personal gerencial de los servicios maternos como los clínicos y personal responsable de la implementación de las recomendaciones en los niveles más directos. Estos últimos fueron seleccionados debido a la importancia de la representatividad que se necesitaba en el estudio de quienes están a cargo de implementar políticas de salud.

Se abordó un análisis cualitativo relacionado con la perspectiva y la opinión de actores clave, organizado en la dimensión de aceptabilidad, adopción, barreras, facilitadores y determinantes del sistema de salud. Estas dimensiones se centraron en varias categorías de análisis que incluyeron: el grado de difusión de los documentos, la calidad del proceso de formulación de las GPC y los protocolos, el nivel profesional, el año de formación profesional, el liderazgo, la percepción de la calidad de los documentos, los factores del sistema de salud que influyen sobre la adopción y las barreras presentadas.

El detalle del contenido de las entrevistas, los grupos de discusión, las dimensiones abordadas y la aplicación en terreno de los instrumentos fueron realizados por tres investigadores independientes, quienes tuvieron una inducción sobre el tema de estudio y fueron calibrados en los instrumentos.

El análisis de las transcripciones de entrevistas y grupos focales se realizó de manera manual y mediante el software Atlas $\mathrm{ti}^{\circledR}$, desde un marco de análisis de contenido con la categorización y la codificación de la información según las dimensiones propuestas originalmente en el diseño de los instrumentos y con la inclusión de categorías emergentes cuando fuera pertinente.

Este estudio recibió aprobación por parte del Consejo Nacional de Bioética en salud de la República Dominicana (Conabios) y del Comité de Revisión Ética de la OPS (PAHOERC). Se diseñó un proceso de consentimiento informado estándar, por el cual cada sujeto de estudio fue informado, consultado y aceptó participar. El consentimiento fue digitalizado y resguardado.

Este estudio contó con el apoyo de la Escuela de Salud Pública de México y de la OPS para el desarrollo de la investigación.

\section{RESULTADOS}

Se recopiló información de cinco centros del ámbito materno-infantil (la Maternidad Nuestra Señora de Alta gracia, la Maternidad De Los Mina, la Maternidad René Klan, la Maternidad Juan Pablo Pina de San Cristóbal y la Maternidad Jaime Sánchez). Los participantes se distribuyeron en cuatro grupos focales. En el mismo período se recogió la perspectiva de los 70 prestadores.

\section{Características de los actores de grupos focales y entrevistados}

La población participante estuvo integrada por 23 enfermeras, 12 médicos residentes de ginecología y obstetricia, 12 médicos especialistas en ginecobstetricia, 10 gerentes de salud y dos líderes formuladores, con un total de 52 participantes de sexo femenino y 18 de sexo masculino.

\section{Conocimiento, aceptabilidad y adopción}

Dentro del acápite de conocimiento sobre la existencia de los protocolos y GPC para la atención a la mujer con preeclampsia-eclampsia, los participantes mostraron dominio de sus contenidos, que fue más evidente entre los actores con más tiempo en el servicio. El personal médico de los centros docentes mostró mayor aceptabilidad de las GPC. Asimismo, las GPC sobre preeclampsia-eclampsia son más conocidas por los profesionales adscritos a hospitales de enseñanza (tres de los hospitales evaluados). La adopción de estas guías se ve favorecida en estos hospitales.

Los especialistas y residentes en ginecoobstetricia destacaron contenidos considerados clave en dichos documentos técnicos, e hicieron referencia a mayor consulta de las GPC y protocolos que los prestadores de hospitales que no tienen actividad docente.

\section{Facilitadores del proceso de formulación de GPC y protocolos}

En los actores primó la valoración positiva para el desarrollo y actualización de las GPC con la metodología GRADE. Esta percepción se registró con mayor frecuencia en los hospitales con programas de residencias médicas. Se identifica una apertura de los participantes al tema de medicina basada en la evidencia y su valoración positiva como herramienta base de la formulación de GPC y protocolos.

La posición de estar de acuerdo con la aplicación de las GPC y los protocolos de atención a la mujer con preeclampsiaeclampsia primó entre el personal de enfermería y el personal médico de los cinco hospitales. En ocasiones, esta posición obedecía a motivos de índole legal. Parte de los actores perciben las GPC y protocolos como piezas regulatorias provenientes de la autoridad sanitaria que deben ser aplicadas de forma obligatoria. Al tratarse de reglas coercitivas, se ve motorizada su adopción.

\section{Barreras percibidas ante el proceso de formulación}

Al profundizar en la temática, surgieron comentarios que destacan la coexistencia de profesionales más jóvenes y médicos especialistas, con más tiempo en ejercicio y que, a la vez, son líderes de opinión y docentes, que muestran resistencia a asumir este tipo de atención sanitaria y al uso de las recomendaciones basadas en evidencias. Un médico obstetra, participante en un grupo focal, refirió que: "Hay resistencia muchas veces del personal con mayor tiempo de servicio que toma como parámetro que: lo que yo hacía es válido, lo sigo haciendo". 
Hubo consenso en las opiniones recabadas en los grupos focales, tanto de las enfermeras como del personal médico, en cuanto a que existen limitaciones para cumplir con las normas y aplicar las recomendaciones basadas en evidencias que plantea las GPC y los protocolos para la atención de la mujer con preeclampsia-eclampsia, sobre todo por la falta de medicamentos, insumos y equipos requeridos. Se hizo hincapié en que, como consecuencia de estas limitaciones, se recurre a prácticas que se institucionalizan en los hospitales.

Un elemento para resaltar que estuvo presente en los hospitales y en las entrevistas a los actores claves del sistema de salud fue la necesidad de generar evidencias nacionales en el abordaje de la preeclampsia-eclampsia, que pueda, a futuro, servir de base a las recomendaciones de las GPC y protocolos nacionales, dado que en la actualidad están basados solo en evidencias internacionales.

En cuanto a desacuerdos para la adopción de GPC y protocolos por razones ideológicas-religiosas, solo en uno de los hospitales se identificó una posición en desacuerdo con base en razones de tipo religiosas, relacionadas con la indicación de aborto terapéutico en pacientes con preeclampsia grave en estadios tempranos de la gestación.

Es importante indicar que, en función de la opinión de los gestores entrevistados, la implementación de GPC y protocolos constituye un desafío impostergable para el sistema de salud dominicano y que es un aspecto para tener en cuenta en todos los documentos normativos.

Los gerentes tienen la percepción de que el sistema de salud dispone de las capacidades administrativas para la implementación de las GPC. Perciben también, que es necesario avanzar en el fortalecimiento institucional del proceso de implementación de GPC, como avanzar hacia un programa nacional de guías y protocolos que pueda asumir los desafíos identificados en la presente investigación. La percepción de los líderes formuladores se relacionó con las fortalezas derivadas de las capacitaciones en metodología GRADE y la integración de asesores expertos clínicos y metodólogos al proceso de producción e implementación de las GPC.

Con respecto a la difusión, los participantes han mostrado discrepancia. Mientras que algunos muestran preferencia por el formato digital, sobre todo las aplicaciones para dispositivos móviles, otro grupo manifiesta nunca haber accedido de esta manera y utilizar el formato físico. Ambos grupos coinciden en la utilidad del formato resumido.

De la misma forma, un grupo considera que la divulgación ha sido suficiente mientras que otro grupo dice que debe reforzarse. Este último grupo sugiere la utilización de medios de comunicación masivos (redes sociales y televisión en hospitales), así como la incorporación de las GPC y los protocolos en los procesos académicos de grado y posgrado en las universidades. Esto incluye las carreras de salud afines (medicina, enfermería, licenciatura en obstetricia) y los programas de residencias médicas (ginecología y obstetricia, emergentología y medicina familiar y comunitaria).

Lo mismo aplica para la consideración sobre la necesidad y beneficio de socializar las guías entre las pacientes. Un grupo manifiesta que no debe hacerse mientras que otros abogan por su implementación. Este último grupo sugiere un modelo adaptado que sea más simple y comprensible para las pacientes y usuarias.

En relación con la implementación, los participantes indican que es necesario fortalecer los mecanismos institucionales que motiven y obliguen a quienes están involucrados en el proceso de atención a la implementación de las GPC y los protocolos. Dentro de las sugerencias indicadas se hallan fortalecer el liderazgo de los directores de hospitales y jefes de servicio; implementar equipos intrahospitalarios de seguimiento a la implementación de las GPC y los protocolos, con su plan adecuado de mejora continua; determinar un régimen de consecuencias ante el no cumplimiento de las GPC y los protocolos; y el diseño de medidas preventivas para la no observación o incumplimiento de las recomendaciones contenidas en las GPC.

\section{DISCUSIÓN}

Los integrantes del sistema de salud dominicano reconocen la metodología GRADE como un instrumento apropiado para la formulación de las GPC. Las barreras que se destacan son la antigüedad en el servicio y no estar adscritos a hospitales docentes. Los resultados de la presente investigación ofrecen recursos para impulsar la política de expansión de la estructura de formulación de GPC a nivel nacional, en el marco de un programa nacional que apoye las competencias de los recursos humanos y los recursos metodológicos para la producción de documentos informados con base en la mejor evidencia disponible. En la República Dominicana se han experimentado cambios de orden cualitativos con la incorporación de recursos metodológicos para la formulación de guías GRADE que nos sitúan en la ruta que posibilita la implementación de un programa nacional de guías clínicas.

Entre los desafíos futuros de los sistemas de salud, se destaca la necesidad de contar con instrumentos de referencia informados por investigaciones contextualizadas y reproducibles, fundamentados en las evidencias que, a su vez, apoyan políticas, programas e intervenciones para impulsar su implementación.

Diversas corrientes del mundo sanitario promueven el desarrollo de modelos innovadores de investigación para evaluar la implementación de políticas y programas con la participación de los responsables de la gestión pública y los tomadores de decisiones que impactan en los sistemas de salud (7).

Los resultados de nuestro estudio exponen la necesidad de fortalecer estrategias que ayuden a cerrar la brecha entre la investigación y la política pública, centrándose en gran medida en la traducción, síntesis e intercambio de conocimientos. Nuevas vertientes fundamentan la investigación en priorizar la atención a los usuarios, incluidos los pacientes, los proveedores, los encargados de formular políticas y los tomadores de decisiones en el sistema de salud (9). Existe un consenso cada vez mayor sobre la importancia de involucrar a las partes interesadas, sobre la premisa de que el diseño conjunto y la coproducción de la investigación tienen el potencial de mejorar la alineación de los procesos de ciencia y política y así reducir el desperdicio de la investigación. La investigación que evalúa la implementación de programas se utiliza cada vez más para identificar problemas comunes en el proceso de implementación y barreras clave que influyen en el acceso eficiente a las intervenciones de salud. Esto último se respalda en los resultados obtenidos en esta investigación, en la que los usuarios y los formuladores de políticas identificaron las principales brechas que obstaculizan la implementación completa de las GPC y de los protocolos de atención relacionados con preeclampsia-eclampsia en el país.

Los resultados de nuestro estudio aplican para la realidad dominicana; las investigaciones desarrolladas a propósito de 
intervenciones de implementación de GPC concluyen en que no existe una única estrategia clara y que el efecto resultante es pequeño $(10,11)$. Estos hallazgos motivan la búsqueda de estrategias nuevas que, enfocadas en producir cambios institucionales en términos de estructura de servicios e instrumentación de las guías, puedan causar impacto sin perder de vista los factores individuales que caracterizan la percepción, los conocimientos y las prácticas de los prestadores de la atención. En términos de experiencia en América Latina, el modelo de programa de GPC de Chile parte de la investigación en implementación que identificó las necesidades relacionadas con documentos que definen los recursos metodológicos, las funciones de los actores, el papel de las academias de educación superior, la coordinación con instituciones formuladoras y una estrategia de implementación (9).

La percepción de que la metodología GRADE es una herramienta apropiada $y$, al mismo tiempo, compleja constituye un desafío para la comprensión de la estructura técnica de las GPC. Se identifica como positiva la participación de líderes formuladores de diferentes sectores en el proceso; en especial el liderazgo del Ministerio de Salud Púbica en la elaboración de las GPC y los protocolos, y la autoridad de los miembros de los hospitales, así como también las sociedades especializadas.

Se destaca la colaboración técnica de la OPS como parte importante de los procesos de implementación y seguimiento del desarrollo de las GPC en el país. Sin embargo, se sugiere aumentar la participación de los actores de las provincias, así como también lograr una participación más activa de parte de sectores de los profesionales independientes, las academias, los investigadores e instancias del sistema de salud. Un aspecto para destacar es la percepción de los participantes en relación con las limitaciones financieras y tecnológicas del sistema para asumir las recomendaciones de las GPC.

Financiación. La Alianza de Investigación en Políticas y Sis-temas de Salud de la Organización Mundial de Salud (OMS), junto con la Organización Panamericana de la Salud (OPS) otor-garon subsidios para la ejecución de una investigación sobre la implementación de programas de salud varios países de América Latina y el Caribe a través del programa iPIER (Impro-ving Program Implementation through Embedded Research). Este estudio fue declarado ganador de la beca en la República Dominicana.

Contribución de los autores. AdlR contribuyó en el diseño, análisis de datos, interpretación y redacción de los primeros borradores y posteriores del documento); JM apoyó en el diseño, análisis de datos, interpretación y redacción del primer y subse-cuente borrador del documento; IB, DFM y MT contribuyeron al proceso de recolección de datos; $\mathrm{MPH}$ y AR contribuyeron con la revisión final de este artículo.

Conflicto de intereses. Ninguno declarado por los autores.

Declaración. Las opiniones expresadas en este manuscrito son responsabilidad del autor y no reflejan necesariamente los crite-rios ni la política de la RPSP/PAJPH y/o de la OPS.

\section{REFERENCIAS}

1. Institute of Medicine. Finding what works in health care: standards for systematic reviews. Washington, D.C.: The National Academies Press; 2011.

2. Organización Panamericana de la Salud. Directriz para el fortalecimiento de los programas nacionales de guías informadas por la evidencia: una herramienta para la adaptación e implementación de guías en las Américas. Washington D.C.: OPS; 2018.

3. Organización Mundial de la Salud. Manual para la elaboración de directrices. Ginebra: OMS; 2015.

4. Ministerio de Salud Pública, Dirección de Epidemiología, SINAVE, República Dominicana; 2017.

5. Gobierno de República Dominicana. Ley General de Salud N. ${ }^{\circ}$ 42-01, promulgada el 8 de marzo del 2001. Disponible en: http:// www.sisalril.gov.do/mobile/pdf/leyes/ley_no_42-01.pdf

6. Gobierno de República Dominicana. Ley N. ${ }^{\circ} 87-01$ que crea El Sistema Dominicano de Seguridad Social, promulgada el 9 de mayo del 2001. Disponible en: http://www.sisalril.gov.do/pdf/leyes/ ley_no_87-01.pdf

7. Langlois EV, Mancuso A, Elias V, Reveiz L. Embedding implementation research to enhance health policy and systems: a multi-country analysis from ten settings in Latin America and the Caribbean. Health Res Policy Sy 2019;17(1):85.

8. Organización Panamericana de la Salud. 53.o Consejo Directivo 66.a Sesión del Comité Regional de la OMS para las
Américas. Washington, D.C., del 29 de septiembre al 3 de octubre del 2014.

9. Herrera P, Fajreldin V, Rodríguez MF, Kraemer P, Mendoza C, Pineda I, et al. Guías de práctica clínica: estudio cualitativo sobre su implementación en los sistemas de salud. Rev Panam Salud Publica. 2017;41:e67.10.

10. Gagliardi AR, Brouwers MC. Integrating guideline development and implementation: analysis of guideline development manual instructions for generating implementation advice. Implementation Science. 2012;7:67.

11. Zamora G, Meneses D, De-Regil LM, Neufeld L, Pena-Rosas JP, Sinisterra OT. Considerations on the development of nutritionrelated guidelines by the World Health Organization and their implementation. Arch Latinoam Nutr. 2015;65(1):1-11.

Manuscrito recibido el 29 de junio de 2020. Aceptado para su publicación, tras revisión, el 6 de octubre de 2020. 


\section{Acceptability and adoption of clinical practice guidelines and treatment protocols on preeclampsia/eclampsia in the Dominican Republic}

ABSTRACT Objective. Estimate the acceptability and adoption by health care workers of clinical practice guidelines and treatment protocols for women with preeclampsia/eclampsia and identify the facilitating factors and barriers to their implementation.

Methods. A qualitative study was conducted, using semi-structured interviews and focus groups in five maternity hospitals. Interviews were compiled for analysis, and barriers and facilitators were characterized.

Results. Seventy health professionals (52 female and 18 male) participated, representing different levels of the health system. The majority of workers and managers were aware of the existence and content of clinical practice guidelines (CPGs) for eclampsia/preeclampsia, especially the participants with more time in the health service. With respect to facilitating factors, both medical and nursing staff were positive about continued development and implementation of high-quality CPGs. There was consensus that limitations exist, especially with respect to a lack of the necessary medicines, supplies, and equipment to meet and implement the established recommendations.

Discussion. The results of the study show the need to strengthen strategies that help close the gap between research and public policy. Studies suggest that research should focus on users, policy-makers, and decision-makers in the health system. The actors in the Dominican health system recognize the GRADE methodology as an appropriate instrument for the development and implementation of CPGs. Implementation barriers require systemic and comprehensive approaches.

Keywords $\quad$ Practice guideline; protocols; patient acceptance of health care; health policy; Dominican Republic.

\section{Aceitabilidade e adoção de diretrizes de prática clínica e protocolos de atenção para pré-eclâmpsia e eclâmpsia na República Dominicana}

RESUMO Objetivo. Estimar a aceitabilidade e a adoção de diretrizes de prática clínica (DPCs) e protocolos de atenção para mulheres com pré-eclâmpsia e eclâmpsia por profissionais da saúde e identificar os fatores facilitadores e barreiras à sua implementação.

Métodos. Desenvolvemos um estudo qualitativo baseado em entrevistas semiestruturadas e grupos focais em cinco maternidades. As entrevistas foram coletadas para análise, sendo caracterizadas as barreiras e fatores facilitadores.

Resultados. $O$ estudo contou com a participação de 70 profissionais da saúde (52 mulheres e 18 homens) que trabalham em diferentes níveis do sistema de saúde. Em sua maioria, os profissionais e administradores estão cientes da existência de DPCs para pré-eclâmpsia e eclâmpsia e conhecem seu conteúdo, especialmente os que têm mais tempo de experiência. Em relação aos fatores facilitadores, os profissionais médicos e de enfermagem consideraram positivo o processo de elaboração e implementação de DPCs de alta qualidade. Houve consenso sobre a existência de limitações, especialmente no que diz respeito à falta de medicamentos, insumos e equipamentos necessários para cumprir e implementar as recomendações.

Discussão. Os resultados do estudo deixam clara a necessidade de reforçar as estratégias que ajudam a estabelecer vínculos entre a pesquisa e as políticas públicas. A pesquisa futura deve priorizar a atenção aos usuários e o apoio aos decisores e responsáveis pela elaboração de políticas no sistema de saúde. Os atores do sistema de saúde dominicano reconhecem a metodologia GRADE como um instrumento apropriado para a formulação e implementação de DPCs. As barreiras à implementação exigem abordagens sistêmicas e abrangentes.

Palavras-chave Guia de prática clínica; protocolos; aceitação pelo paciente de cuidados de saúde; política de saúde; República Dominicana. 